\title{
Observation of Vibronic Emission Spectrum of the Jet-Cooled $m$-Cyanobenzyl Radical in a Corona Excitation
}

\author{
Gi Woo Lee, Jong Keun Park, and Sang Kuk Lee \\ Department of Chemistrv and Chemistry Institute for Functional Materials, Pusan National Cniversit, Pusan 609-735, Korea \\ Received Januarv 7, 2004
}

Key Words : Cyanobenzyl radical. Spectroscopy

Transient species such as molecular ions and molecular radicals are of considerable interest in chemistry because they are believed to play an important role in chemical reaction as intermediates. Many techuiques have been developed to find an ideal enviromment suitable to produce and preserve the radicals for the spectroscopic work. ${ }^{\text {lof }}$ Of the methods employed. supersonic jet expansion with a large amount of inert carrier gases has been proved to be effective in the gas phase. However the nature of high reactivity and short lifetime of free radicals has certainly slowed their experimental study in free jet.

Among mono-substituted benzyl radicals. " the xylyl radicals have been extensively studied by various methods. The assignments of vibronic bands observed in the visible region have been carried out to determine the vibrational mode frequencies in the ground electronic state. Also. the torsional contribution of the methyl rotor has been obtained from analysis of the rotational contours of the high resolution Fourier transform emission spectra

As for halogen-substituted benzyl-type radicals the vibrational modes of fluorobenzyl radicals have been determined using different spectroscopic techniques such as laser induced fluorescence (LIF) ${ }^{67}$ and coronal excitation. ${ }^{8-10}$ Weakly emitting chlorobenzyl radicals have been recently identified from the vibronic emission spectra. ${ }^{11-13}$ in which the sequence of electronic energy of the lowest excited electronic state shows a similar tendency to the chlorotoluenes.

Cyanobenzyl radicals are interesting species in spectroscopy. The analysis of the vibronic bandshape of the $p$ cyanobenzyl radical has revealed that the lowest excited electronic state is $2^{-} \mathrm{B}_{2}$ which is different from other types of benzyl radicals. ${ }^{14}$ Also. several vibrational modes of the $p$ isomer in the ground electronic state have been determined from the analysis of vibronic emission spectrum observed. ${ }^{15}$ However. other isomers of cyanobenzyl radicals have not been reported in any spectral region due to the weak intensity of fluorescence and less symmetry.

In this work. we report for the first time the observation of the vibronic emission spectrum of the $m$-cyanobenzyll radical that was generated from the $m$-tolunitrile seeded in a large amont of inert carrier gas He using a pinhole type glass nozzle in a corona excitation supersonic expansion. The

"Corresponding Author. Fax: +82-51-516-7421, e-mail: skleeta pusan.ac.kr lowest electronic transition from the origin band of the visible emission spectrum observed has been measured and compared with those of other isomers.

The formation and excitation of the $m$-cyanobenzyl radical in the gas phase have been accomplished by using a teclunique of corona excited supersonic expansion. ${ }^{16-20}$ of which the experimental apparatus is similar to that described elsewhere ${ }^{21}$ The $m$-cyanobenzyl radical was produced and vibronically excited in a jet from $m$-tolunitrile seeded in a large amount of buffer gas He using a corona excitation. Reagent grade $m$-tolunitrile purchased from Aldrich was evaporated at room temperature under $2.0 \mathrm{~atm}$ of carrier gas in the sample cell. The concentration of the precursor in the carrier gas was adjusted for the maximum emission intensity monitored from the strongest band and believed to be about $1 \%$ of the gas mixture. The gas mixture was expanded through the $0.4 \mathrm{~mm}$ diameter of the pinhole-type glass nozzle made in this laboratory according to the method described previously. 2 The long sharpened stainless steel rod acting as an anode was connected to the high voltage DC power supply in the negative polarity in which the axial discharging current was about $5 \mathrm{~mA}$ at a $1500 \mathrm{~V}$ dc potential and stabilized using a $150 \mathrm{k} \Omega$ current limiting ballast resister.

In the original nozzle developed by Engelking. ${ }^{18}$ the coronal electrode sits just behind the nozzle opening on the high pressure side which leads to excitation before expansion. causing a nozzle-clogging problem when heavy organic compounds were employed as the precursor. Thus, we have made the metal tip of the anode extend through the nozzle to reduce the clogging of the nozzle throat by decomposition products and soot deposits of $m$-tolunitrile. Although less than $1.0 \mathrm{~mm}$ of the metal tip was exposed outside the end of the nozzle capillary, this significantly improved the clogging problem by partially allowing excitation to occur after expansion. $=2$ The modified Engelking-type nozzle has been applied to the generation of many benzyl-type radicals. ${ }^{23}$

The Pyrex expansion chamber was made of thick-walled Pyrex tubes (Chenglass CG-138-02) of $50 \mathrm{~mm}$ in diameter. The chamber was evacuated by a $800 \mathrm{~L} / \mathrm{min}$ mechanical vacuum pump. resulting in the pressure range of $0.5-1.0$ Torr during continuous expansion with $2.0 \mathrm{~atm}$ of backing pressure. The cathode made of a long copper rod was positioned to be parallel to the jet direction under the expansion chamber to buffer the spectrometer from arching noise.

A bright blue-green color in the jet was the evidence of the presence of the $m$-cyanobenzyl radicals. The light emanating 


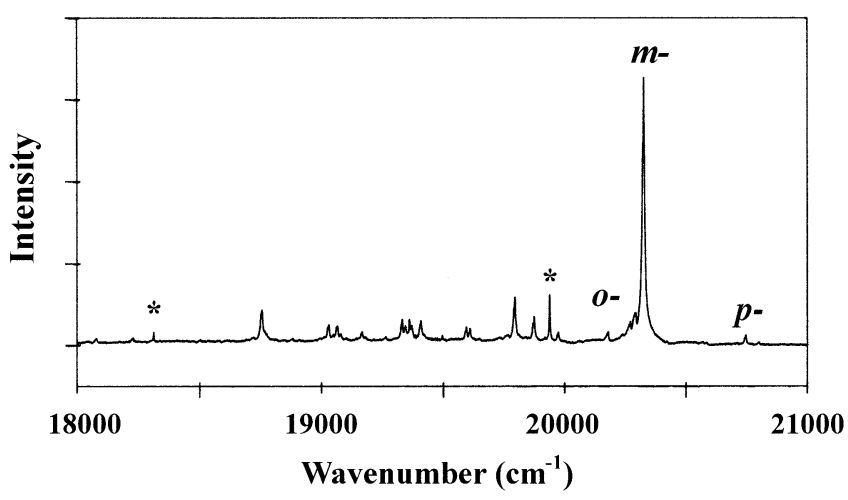

Figure 1. A portion of the vibronic emission spectrum of the jet cooled m-cyanobenzy] radical in the $\mathrm{D}_{1} \rightarrow \mathrm{D}_{0}$ transition. which was generated from m-tolunitrile seeded in a large amount of inert carricer gas lle using a pinhole type glass nozzle in a corona excitation. The peaks marked with an asterisk in the spectrum indicate the He atomic transitions. The weak bands at 20172 and $20744 \mathrm{~cm}^{-1}$ are the origin bands of $\theta$ - and $p$-isomers. respectively. which were also generated from the impurity of the sample in a corona excitation.

from the downstream jet area $5 \mathrm{~mm}$ away from the nozzle opening was collimated by a quartz lens $(f=5.0 \mathrm{~cm}$ ) placed inside the expansion chamber and focussed onto the slit of the monochromator (Jobin Yvon $\mathrm{U} 1000$ ) containing two 1800 lines $/ \mathrm{mm}$ gratings, and detected with a cooled photomultiplier tube (Hamamatsu R649) and a photon counting system. During the scans, the slits were set to $0.100 \mathrm{~mm}$. providing a resolution of about $\mathrm{I} \mathrm{cm}^{-1}$ in the visible region. The spectral region from 18000 to $24000 \mathrm{~cm}^{-1}$ was scanned at the step of $1.0 \mathrm{~cm}^{-1}$ over 2 hrs to obtain the final spectrum shown in Figure 1. The wavenumber of the spectrum was calibrated using the He atomic lines ${ }^{24}$ observed in the same spectral region as the $m$-cyanobenzyl radical and is believed to be accurate within $0.5 \mathrm{~cm}^{-1}$.

In the $m$-cyanobenzyl radical, the electronic interaction between the $\mathrm{CN}$ group and the aromatic ring is undoubtedly of second-order compared to that between the methylene group and the benzene ring since the molecule has a planar structure with seven delocalized $\pi$ electrons. Thus, the $m$ cyanobenzyl radical should exhibit a close relation to the benzyl radical and one may be able to relate the two lowest lying electronic states of the $m$-cyanobenzyl radical to the parental benzyl $2^{2} \mathrm{~B}_{2}\left(\mathrm{D}_{2}\right)$ and $\mathrm{I}^{2} \mathrm{~A}_{2}\left(\mathrm{D}_{1}\right)$ states.

Figure I shows a portion of the vibronically resolved emission spectrum of the $m$-cyanobenzyl radical in the $\mathrm{D}_{1} \rightarrow$ $\mathrm{D}_{0}$ transition in the visible region. Most of the strong bands are observed with an excellent $\mathrm{S} / \mathrm{N}$ in the region of 18500 $20500 \mathrm{~cm}^{-1}$. They consist of a strong origin band of the $D_{1}$ $\rightarrow D_{0}$ transition at $20324.0 \mathrm{~cm}^{-1}$ (in air), followed by a series of vibronic bands at lower energies. Diatomic fragınents such as $\mathrm{CH}$ and $\mathrm{CN}$ radicals, ${ }^{25}$ resulting from the coronal discharge of the precursor have been identified with a weak intensity at 23200 and $23750 \mathrm{~cm}^{-1}$, respectively. The weak sequence bands from $\mathrm{He}_{2}$ at the excited electronic state have been observed in the region of $21400-21600 \mathrm{~cm}^{-1}$ as reported previously. ${ }^{25}$ The origin bands of the 0 - and $p$-isomers are also observed with weak intensity at the frequencies reported. which are believed to be formed from the small amount of impurity in the sample. The absence of bands with noticeable intensity in the blue of the origin confirms the efficient vibrational cooling at the excited electronic state, which is an important characteristic of the pinhole-type glass nozzle. ${ }^{20}$

In summary, the jet-cooled m-cyanobenzyl radical was generated for the first time in a jet from $m$-tolunitrile seeded in a large amount of inert carrier gas $\mathrm{He}$ and vibronically excited using a pinhole-type glass nozzle in a corona excited supersonic expansion. The position of the origin band was measured from the well-resolved visible emission spectra in the $D_{1} \rightarrow D_{0}$ transition. From the comparison with other isomers, it has also been found that the trends are similar to those of chlorobenzyls, xylyls, and tolunitriles.

Acknowledgment. This work was supported by the Korea Research Foundation Grant (KRF-2002-015-CP016I).

\section{References}

1. Ian. X. Q.: Wright. 1. G.: Millcr. I: A. Electronic Spectroscopv of Free Radicals in Supersonic Jets: Jet Spectroscopy and Wolectilar Dinomics: [ lollas, J. M.: Phillip., D.. Hds.: [3lackie Academic \& Prolessional: I ondon. 1994.

2. Langkilde. F. W.: Bajdor. K.: Wilhandt. R.: Negri. F.: Zertelto. F.: Orlandi. G. J. (hem. Phys. 1994. /00. 3503.

3. Solco. J. I.: Carrick. P. G. I. Hol. Spectrose. 1995. 173. 277.

4. Charlton. T. R.: Thrush. B. A. Chem. Phys. Lett 1986. /25. 547.

5. Suh, M. II: I ee, S. K.: Miller. Т. A. J. Hol. Spectrosc, 1999. IOH, 2II. (2) Selco, I. I.: Carrick. P. (i. J. Hol. Spectrosc. 1989, 137. 13

6. Fukushima. M.: Obi. K. J. Chem. Phns. I990. 93.8488.

7. Lin. T.-Y.: Tan. X.-Q.: Corny. T. M.: Williamson. J. M.: Cullin. D. W.: Miller. T, A. Chem. Phys. 1992, 167, 203.

8. Lee. S. K.: Batek. D. Y. Chem. Phys. Iett. 1999. 301.407.

9. I ee, S. K.: Ahn. B. 1: Chem. Phys. Lett. 2000. 32/. 25.

10. Lee. S. K.: Lee. S. K. I. Phys. (Hent A 200I. /05. 3034.

11. Lec. S. K.: Back. D. Y. (Trm. Phys. Letl. 1999. 304.39.

12. Lec. S. K.: Chac. S. Y. J. Jhws (hem. A 2001. /05. 5808.

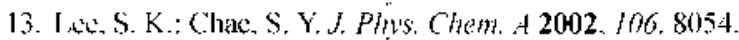

14. I'ukushima. W.: Saito. K.: Obi. K. J. Wol. Spectrose. 1996. 180. 389.

15. Lee. S. K.: Ahrl. B. L: (hem. Phys. Lett 2000. 32f. $60 \mathrm{l}$.

16. Smallcy. R. E.: Wharton. L.: Levy. D. H. J. Chem. Phs. 1975. 63. 4977.

17. Demtroder. W. Laser Spectroscopy: Springer-Ver]ag: Berlin. Cermany, 1980 .

18. Droege. A. I.: Ingelking. P. C. (Them. Phis. Lett. 1983. 96. 316.

19. Engelking. P. C. Rev: Sci. Instrum. 1986. 57. 2274.

20. Lec. S. K.: Chac. S. Y. J. Ihys (Them. A 2003. /07. 6554.

21. Han. M. S.: Choi. I. S.: Lec. S. K. Bult Korean Chem. Soc. 1996. 17.991 .

22. I tee. S. K. Chent. Ph's. Left. 2002, 358. 110.

23. Chale. S. Y.: I.ee. S. K.: Kim. S.-H I. Bull. Norean (hem. Soc 2002. 23. 795 .

24. Wiese. M, 1.,: Smith, M, W: Glconnon. B, V. Atomic Titurition Frobubitities. NSRD-VBS4: 1966.

25. Pearse. R. W. B.: Gaydon. A. G. The demification of Wotechlar Sipectro, 5th ed.: Chapman and I [all: I sondor]. (jreal I3ritain. 1976.

26. L eee. S. K.: Chate, S. Y. Chem. Phy 2002. 284.625.

27. Fujii. M.: Yamauchi. M.: Jakazawa. K.: I10. M. Spectrochimica fcta 1994. 50.4. 1421.

28. I ce. S. K, to be published. 